\title{
New Contribution for Theoretical and Experimental Investigation for Evaluation of Transmission Line Relays Using Advanced Tools
}

\author{
H. Saber Ebrahim 1, A.M. Karim Eldin 2, S. S.Dessouky 3
}

\begin{abstract}
This paper presents a technique for testing protection relays using advanced simulation tools and lab implementation models for a transmission line. Different Scenario were simulation and implemented on the transmission line in the lab the output voltage and current waves for the two process were filtered using digital filters to remove the transient components. Then fed to the programs describe the function of different protective relays. These evaluate the performance of protective relays for both the signals. Obtained from the lab model and the signals obtained from the simulations. The simulation were performed using Alternative Transients Program (ATP). The programs operate correctly for fault cases obtained by lab model simulator.
\end{abstract}

Keywords: protective relays, power system protection, transmission line.

\section{I- INTRODUCTION}

Relay testing is a very important issue when applying the protective relays. Vendors need an evaluation tool to validate the design of the relay logic and communication. Utilities need a tool to compare the performance of different relays, calibrate relay settings and perform troubleshooting. Operating time of numerical relay is a random value. It is necessary to perform a large number of tests, to determine statistical properties of the relay responses to check its selectivity and average tripping time. Problem definition why and when the transient tests are needed? What procedures should be applied in transient testing? How the transient tests should be created and implemented that will improve transient testing [1]? .

${ }^{1}$ Suez University, Faculty of industrial education, Dept. of Electrical Power and Machines, Suez, Egypt.

${ }^{2}$ Port Said University, Faculty of engineering, Dept.of Electrical Power and Machines, Port Said, Egypt

${ }^{3}$ Port Said University, Faculty of engineering, Dept.of Electrical Power and Machines, Port Said, Egypt.
Histories of relay testing the first relays were electro- mechanical relays. These devices were quite simple compared to today's relays and so were the test devices and the test methods for testing over current protection. due to the fact that the relays become more and more complex with some devices having more than 2000 settings, traditional test methods are no longer appropriate. The relay functions are very advanced and also use complex models of the network for calculating. The testing side has to adapt its methods to be able to assess the relays functionality [2]. The main target of these techniques important goal: to check selectivity of protective relay and average speed of operation. Although the shape of the operating characteristic is not explicitly verified in transient testing, the main functional purposes of the operating characteristic to provide selectivity, reliability, sensitivity and satisfactory speed of operation implicitly checked in transient testing. It is usual practice to verify the operating characteristic by testing. Shape of the operating characteristic is defined for steady state values of currents and voltages. Classical approach in relay testing with phasors of currents and voltages is used to verify the operating characteristics [3]. 


\section{II- EVOLUTION OF RELAY TESTING}

This section will outline the evolution of relay testing to better understand the choices available to the relay tester when testing modern digital relays several concepts are realized in practice to conduct transient testing. These concepts include application of various hardware and software tools to create test cases and generate transient waveforms. Theoretical considerations of transient testing and its application with numerical relays are not well understood. Existing concepts in transient testing are focused on testing tools application with random and intuitive definition of test scenarios. Various types of microprocessor relays are tested and evaluated through the set of scenarios [4].

In order to analyse the operation of the protection system during induced fault testing in the croatian power system, a simulation using the computer-aided protection engineering (CAPE) software has been performed. Once the accuracy of the simulation model had been confirmed, a series of simulations were performed in order to obtain the optimal fault location to test the protection system. Results were used to specify the test sequence definitions for the simulation end-to- end relay testing using advanced testing equipment with Global Positioning System (GPS) synchronization for secondary injection in protection schemes based on communication [5].A method for improving the quality of tests and the ability to derive meaningful analysis data is discussed Power system modeling and test procedure for using modern digital simulators are presented [6].

Methods for on-line testing of analog and digital measurement channels of protection relays have been presented during the test, all protection functions of the device are active like during the normal work. Moreover no

special hardware is installed inside the protection relay.

The methods enable to reveal subtle changes in the measurement channel circuitry frequency characteristic [7]. The design of new relay performance testing scheme, especially for complete digital relays is presented.

\section{III- SIMULATION PROCEDURE}

1- Generate test cases. The ATP model of power network and batch simulation program are used to generate test cases which contain transient voltage and current waveforms related to various disturbance scenarios.

2- Convert Data format. A program developed automatically converts various formats of test cases to the format which can be recognized by Relay Assistant software.

3- Create test session. The test session is created by loading selected test cases with each test session contains specific scenarios sorted by different types of disturbances or power system operating conditions. For example, the fault session can be sorted with fault type, location, inception angle and resistance.

4- Set protective relays. The relay settings group corresponding to a given transmission line and protection scheme is activated through the relay setting program.

5- Execute test and retrieve report. The "real" voltage and current signals generated by simulator are sent to the relay. The relay responds to the input signals for each case and generates an event report containing the detailed operation information. The trip signals are captured by simulator as output signals and used to automatically calculate the initial operation feature such as tripping time. The event reports are collected by the retrieval program for further study. 


\section{IV-SYSTEM DESCRIPION}

The hardware implementation is required to demonstrate the ability of the on line implementation of the suggested technique in real time. Fig.(2) shows the overall experimental setup. In this figure, the laboratory model consists of digital power system simulator is used to test the over current protective relay and the relay acts to operate the appropriate circuit breakers to prevent damage to personnel and property confirm the simulation results obtained from the simulation tool.

Fig.(1) shows a model by ATP and model which consists of:

1- Generator (220v) 3ph ac

2-Transmission line (transposed line) $300 \mathrm{~km}$

3-Circuit breaker

4- Probe current

5-Switch time three phase $(a, b, c)$

6-Load

7-Ground

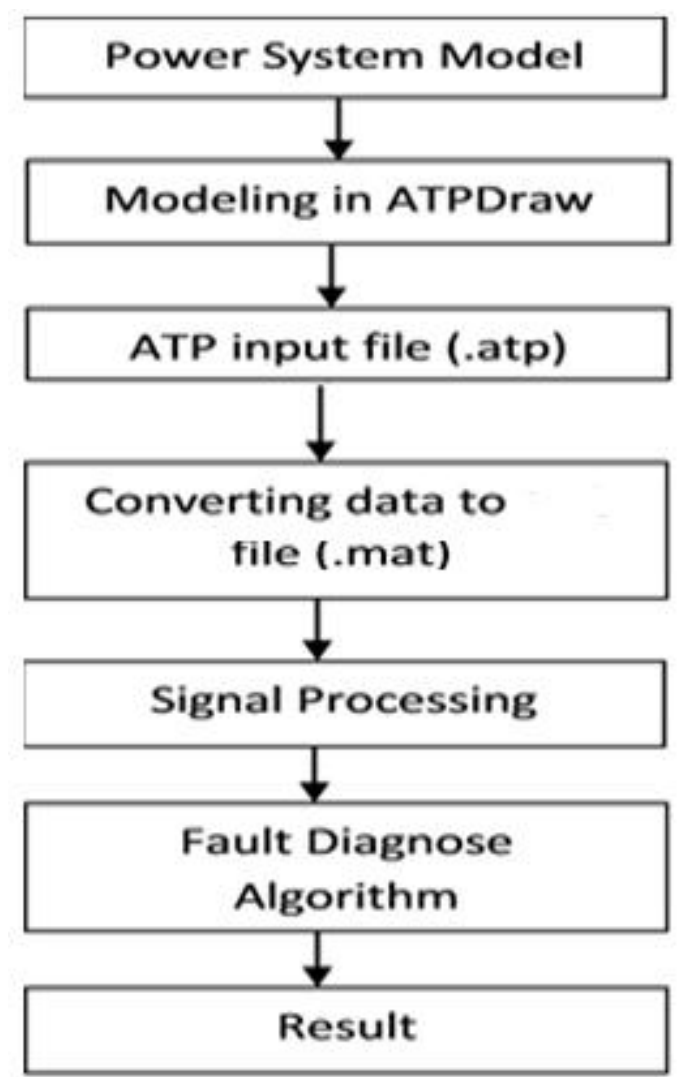

Fig (1) Framework of the simulation tool for test of fault diagnosis algorithm

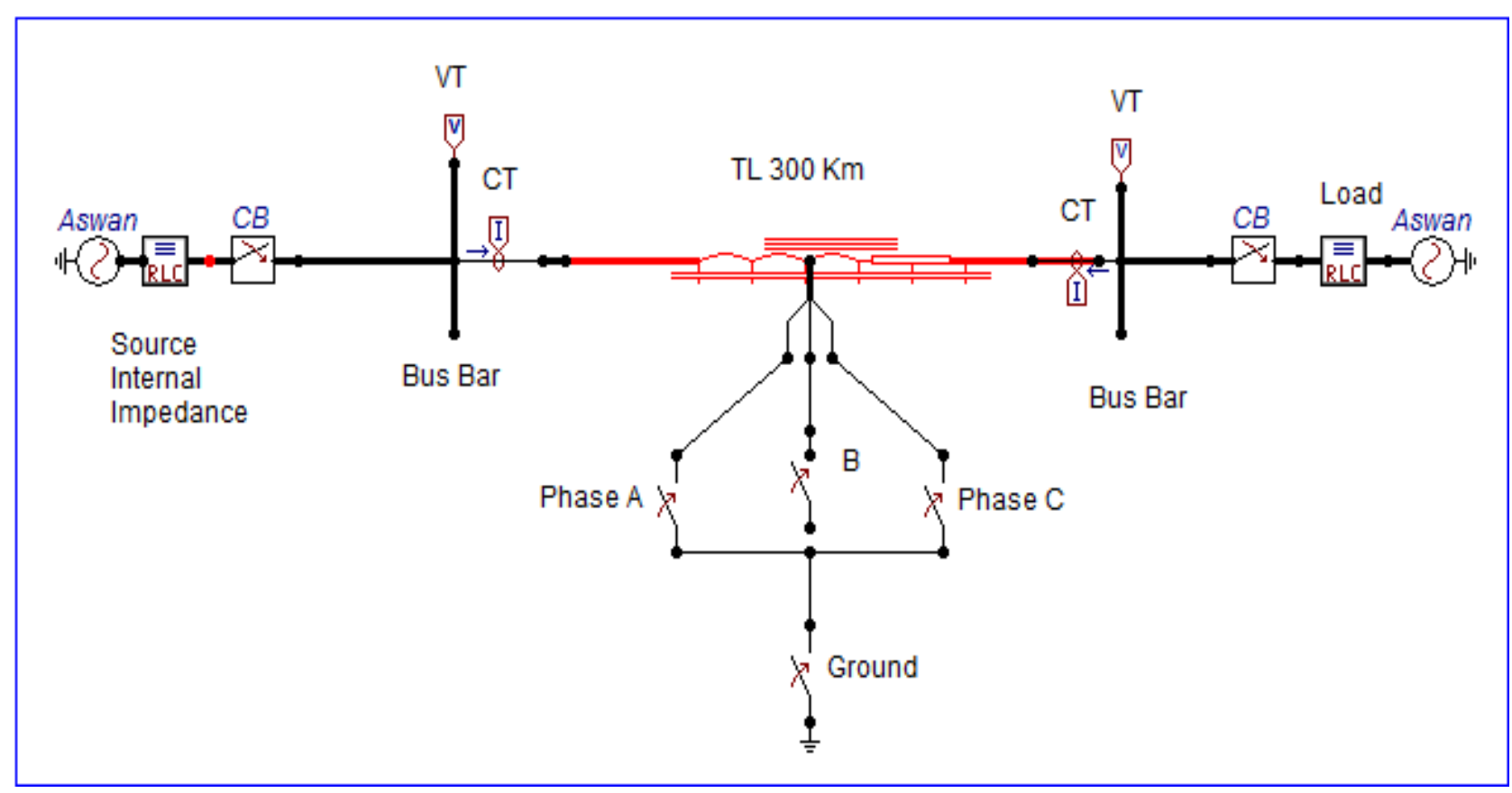

Fig (2) The studied configuration system 


\section{VI- FAULT CASES STUDY BY USING ATP PROGRAM}

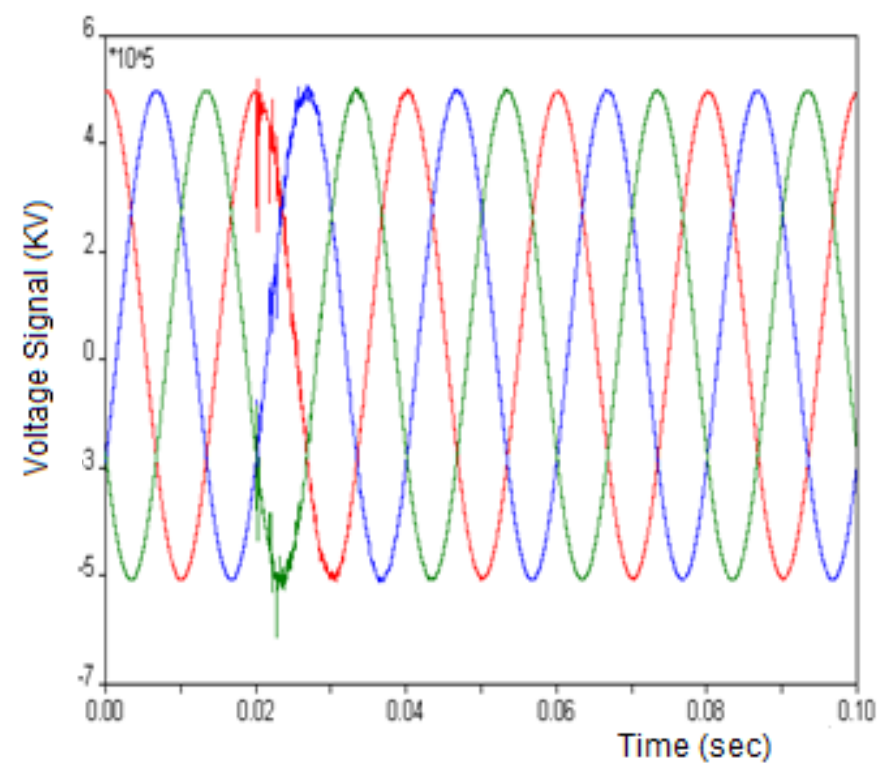

Fig.(3) Three Phase Voltage Signals for A,B

\section{Fault}

The case study of fault shown in fig.(3) indicating a fault at $\mathrm{T}=0.02 \mathrm{~s}$ and voltage $=500$ $\mathrm{kv}$ as a result of over voltage on the a,c phase which led to the occurrence of noise on the signal a,b phases.

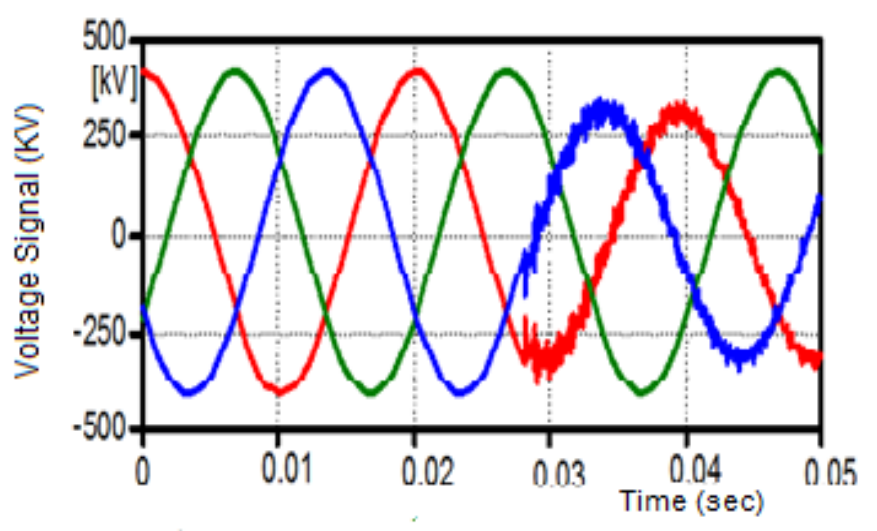

Fig.(5)Three Phase Voltage Signals for a,c, g fault

The case study of fault shown in fig.(5) indicating a fault at $\mathrm{T}=0.04 \mathrm{~s}$ and voltage $=250$ $\mathrm{kv}$ as a result of over voltage on the a,c,g phase which led to the occurrence of noise on the signal a,c,g phases to ground voltage.

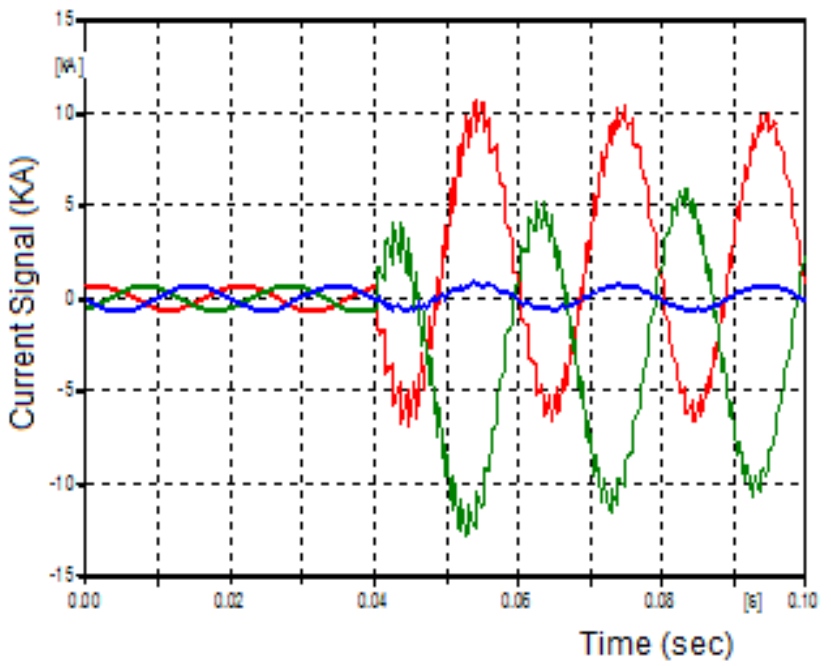

Fig.(4)Three Phase current Signals for A,B, G Fault

(The case study of fault shown in fig.(4 indicating a fault At $\mathrm{T}=0.04 \mathrm{~s}$ and current $=10$ $\mathrm{KA}$ as a result of over current on the $a, b, g$ phase which led to the occurrence of noise on the signal .a,b,g phases to ground current

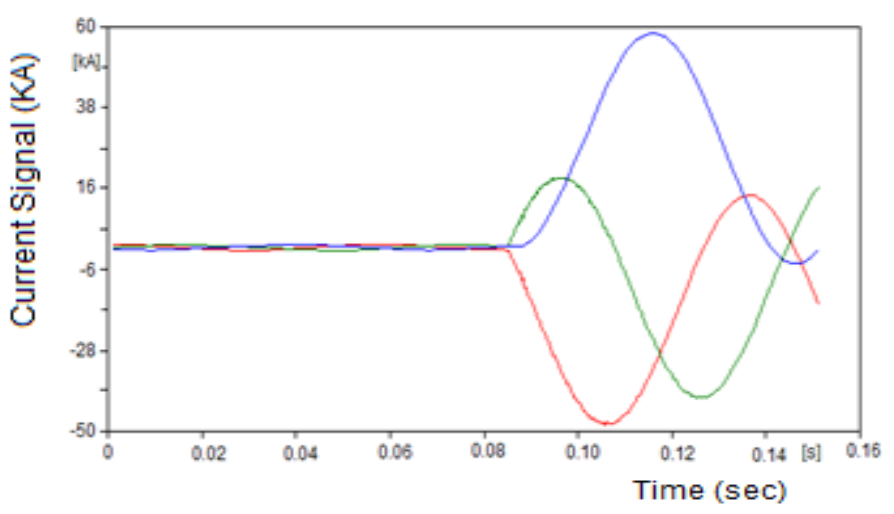

Fig.(6)Three Phase Current Signals for a,c,g Fault

The case study of fault shown in fig.(6) indicating a fault At $\mathrm{T}=0.08 \mathrm{~s}$ and current $=60$ $\mathrm{KA}$ as a result of over voltage on the a,c,g phase which led to the occurrence of noise on the signal a,c,g phases to ground current . 


\section{VII-EXPERIMENTAL SETUP}

The laboratory model in fig 7 consists of one pi section transmission line where their resistance, inductance and capacitance as a lumped parameters for each phase are $3.6 \Omega$, $115 \mathrm{mh}, 150 \mathrm{nf}$. The model is fed from a three phase 380/220 V transformer. A single-phase voltage signals are reduced from the rating of laboratory power source " $220 \mathrm{~V}$ " to a value suitable for the data acquisition card by an ordinary voltage transformer with a ratio of 220/3.the output signals are fed directly to the acquisition card for further processing.

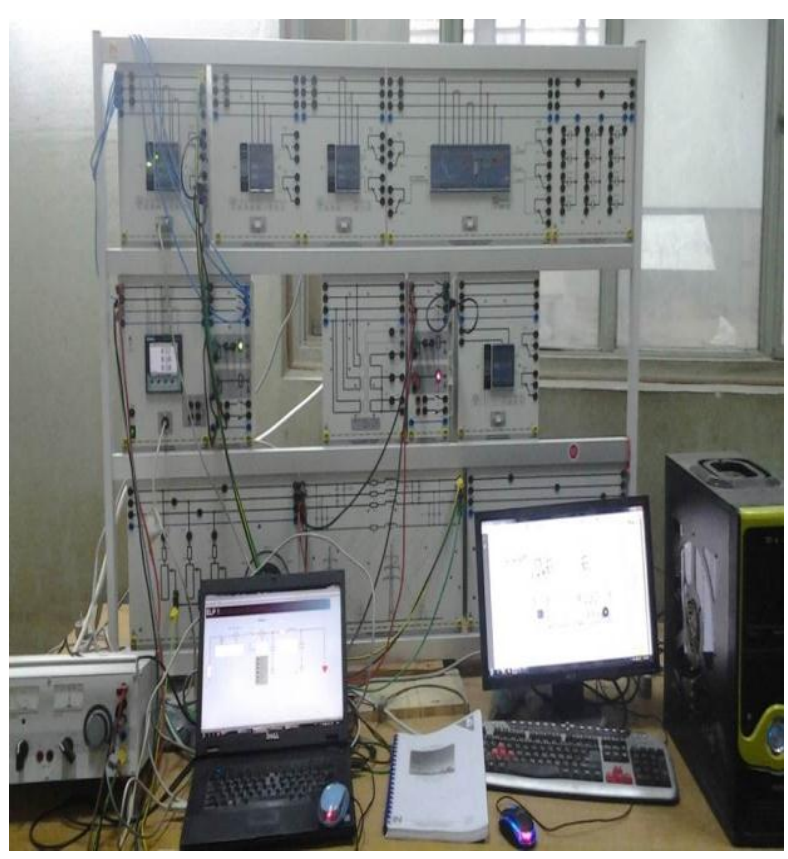

Fig.(7) Lab setup for physical relay test

\section{IX-RESULTS FROM SIMULATOR}

Results from Simulator In the simulation part, an ATP model was designed and tested it, we observed some noise on some signals was found. Conversion of data file to matlab is done to filtrate the signals form noise. In the experimental part: the laboratory model is implemented which compatible with the simulation model with the some data to show the current and voltage signal of the relay which protect the transmission line, the result through interface card then connect it extract to computer with SCADA program stellated on it.

Experimental Results After conducting the test data of cost on the relay on the transmission line noticed errors on the aspects which led to the existence of phases signal emerging and then, transfer the matlab file to filter signal from the noise and at a certain separation of the time has been set the relay upon it when an error is given relay trip signal to the trip occurrence in less than a second time in order to disconnect the power and knowledge of error and treat the cause immediately and to protect the electrical system from damage incurred by and because of the over current and comparison between the output signals and the time of separation in simulation found that there is a match between the theoretical and practical in terms of output signal and the objective is to speed error and remove the damage caused by the overcurrent separation.

\section{X-COMPARISON OF THEORETICAL AND EXPERIMENTAL RESULTS}

After conducting the test data of cost on the relay on the transmission line noticed errors on the aspects which led to the existence of phases signal emerging so, to filter the signal from the noise and at a certain separation of the time has been set the relay upon it when an error is given relay trip signal to the trip occurrence in less than a second time in order to disconnect the power and knowledge of error and treat the cause immediately and to protect the electrical system from damage incurred by and because of the over current and comparison between the output signals and the time of separation in simulation found that there is a match between the theoretical and practical in terms of output signal and the objective is to speed error and remove the damage caused by the overcurrent separation. 
XI-COMPRESSIONBETWEEN

SIMULATION AND EXPERIMENTAL

RESULTS

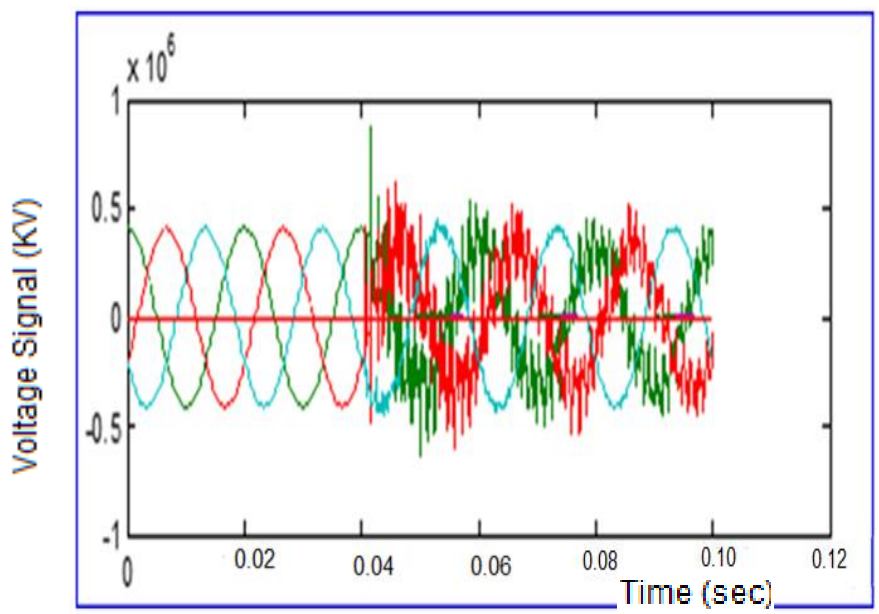

Fig.(8) Experimental Voltage Signal for DLG Fault on Phase A,C

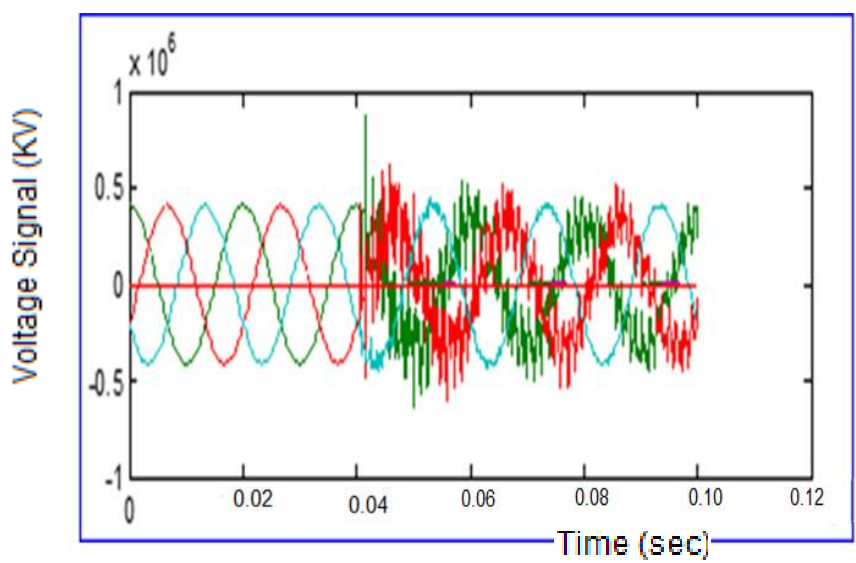

Fig.(9) Digital Simulation of Voltage Signal for DLG Fault on Phase A,C

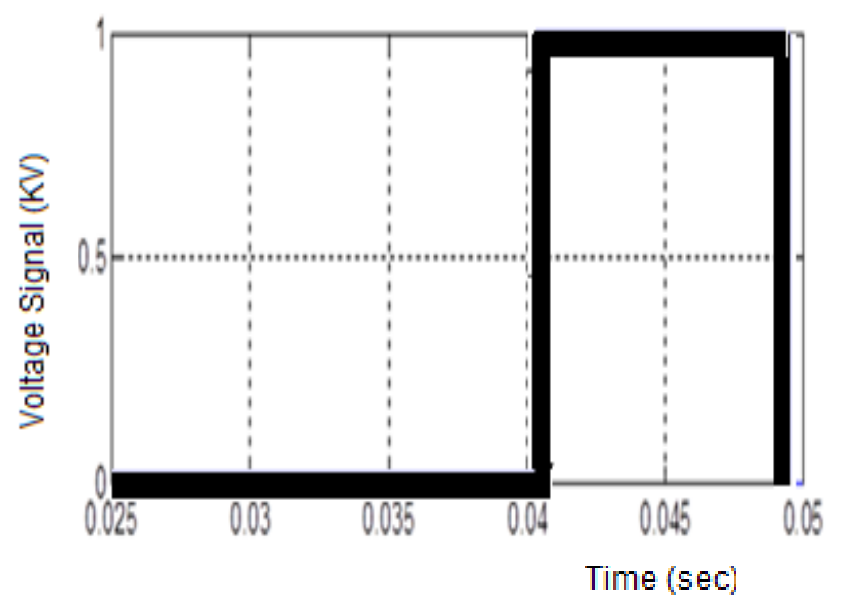

Fig.(10) Relay Operation for DLG Fault on Phase A,C

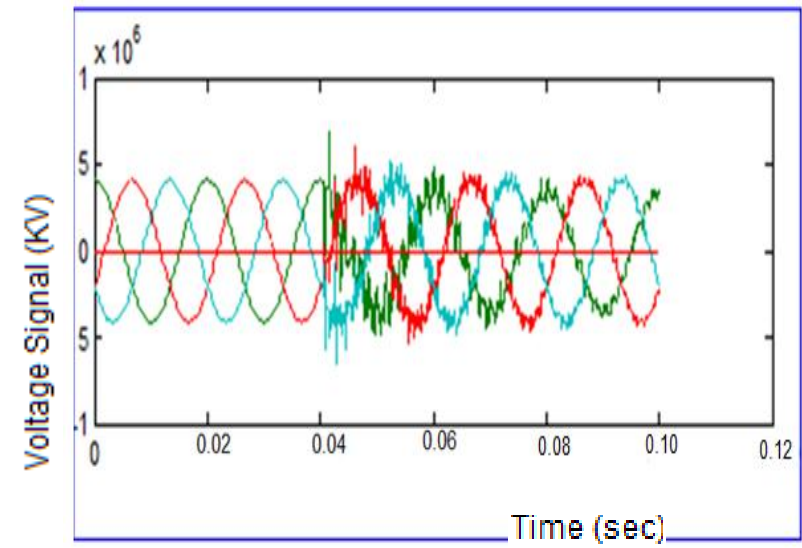

Fig.(11) Experimental Voltage Signal for SLG Fault on Phase C,G

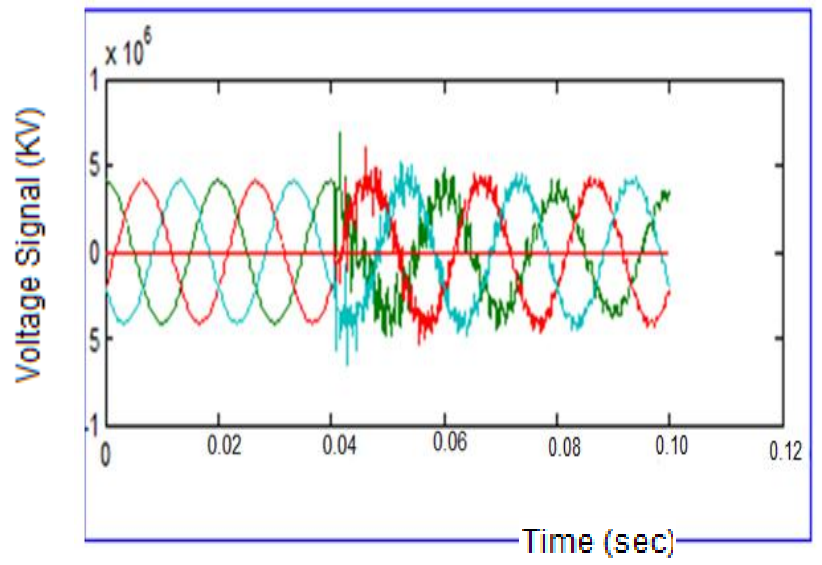

Fig.(12) Digital Simulation of Voltage Signal for SLG Fault on Phase C,G

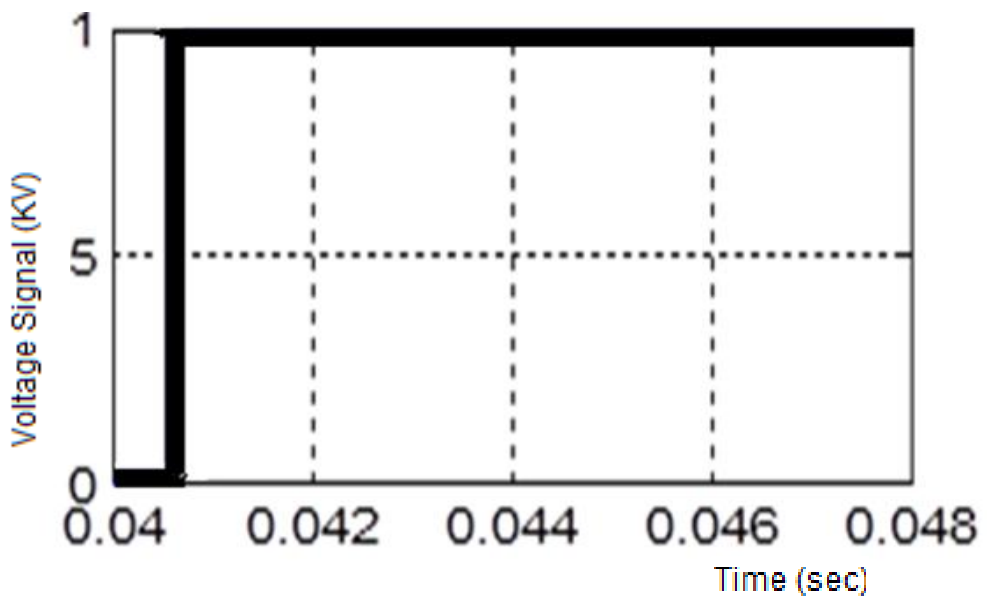

Fig.(13) Relay Operation for SLG Fault on Phase C,G 
- In the study of this case Fig (8) indicating a fault on the c-g phase as a result of over current and compared with the fault located in Fig (9) it turned out that the result of the simulation of the c-g phase is similar to the result of the simulator of the $\mathrm{c}, \mathrm{g}$ phase, since the time and the distance of fault occurrence are similar in both cases and by MATLAB program filtration was done for the signals of $\mathrm{c}, \mathrm{g}$ and $\mathrm{rms}$ in Fig (10) at which the fault occurred and turned out to be $0.04 \mathrm{~s}$ which was to equivalent data sampling 2000S/cycle .

- In the study of this case Fig (11) indicating a fault on the $\mathrm{c}-\mathrm{g}$ phase as a result of over current and compared with the fault located in Fig (12) it turned out that the result of the simulation of the c-g phase is similar to the result of the simulator of the $c, g$ phase, since the time and the distance of fault occurrence are similar in both cases and by MATLAB program filtration was done for the signals of $\mathrm{c}, \mathrm{g}$ and $\mathrm{rms}$ in Fig (13) at which the fault occurred and turned out to be $0.04 \mathrm{~s}$ which was to equivalent data sampling 2000S/cycle.

\section{XII - CONCLUSION}

- The main functional purposes of the operating characteristic to provide selectivity, reliability, sensitivity and satisfactory speed of operation implicitly checked in transient testing. It is usual practice to verify the operating characteristic by testing. Shape of the operating characteristic is defined for steady state values of currents and voltages. classical approach in relay testing with phasors of currents and voltages is used to verify the operating characteristics.
- Defining purpose of transient testing: It was shown theoretically why the transient tests are necessary and when the transient tests are needed.

- Defining test procedures: It was proposed how the transient tests should be defined and conducted to check selectivity and average tripping time of transmission line protective relays.

- Using different test equipment hardware: It was proven that advanced testing could be conducted with digital

- Simulators of high output power in the laboratory, as well as with compact test sets suitable for field application.

- Using different modeling and simulation tools: By applying modeling and simulation tools in relay testing, it was shown how the advantages of different software tools can be utilized to achieve best possible conditions for testing applications.

- The relay evaluation and testing in the future may be made more detailed and precise allowing for further improvements in the performance and reliability of protective relays and relaying.

\section{XIV - REFERENCES}

1. Chris Werstiuk, Manta Test Systems "Testing Requirements for Microprocessor Relays" Valence Electrical Training Services, "M.Sc thesis, royal technical University of Stockholm 2010.

2. D. Ristanovic, "New Methodology for Transmission Line Relay Testing and Evaluation Using Advanced Tools" M.Sc, Electric Power Engineering Texas, University of Technology,Dec. 2003.

3. J. Havelka, R. Malarić, and K. Frlan, "Staged-Fault Testing of Distance Protection Relay Settings", Measurement Science Review, IEEE Power EngineeringSociety General Meeting, pp. 1-4, Volume 12, No.3, 2012. 
4. B.S. Rigby, "Automated real-time simulator testing of protection relays" IEEE Power Engineering Society Conference and Exposition in Africa, Power Africa '07, pp. 1-7, 16-20 July, 2007.

5. A. Nowa kowski, A. Licorice, and Z. Kolo dziejczyk, "Protection Relay Testing in Smart Grids" International Conference on Clean Electrical Power, College Station, Texas , University of Saskatoon 2011.

6- Y. Liu, H. Gao, X. Wei, P. Wei, M. Xiang, and C. Zhou, "Performance Testing of Complete Digital Relays Based on ATP/EMTP" 4th International Conference on Electric Utility,(DRPT), pp. 83-87, 2011.

7- Stefan Schwabe, "New Approach to Simulation Based Type Testing of Protection Relays" International Protection Testing Symposium, $\mathrm{PhD}$ thesis, University of Saskatchewan Canada, pp. 1-7, 2011.

8- Göteborg Sweden "Numerical Distance Protection Relay Commissioning and Testing" M.Sc, Electric Power Engineering Chalmers University of Technology 2005. 